\title{
The association between psychosocial factors and change in lifestyle behaviour following lifestyle advice and information about cardiovascular disease risk
}

\author{
Rebecca A Dennison ${ }^{1 *}$ D, Adina L Feldman², Juliet A Usher-Smith ${ }^{1}$ and Simon J Griffin ${ }^{1,2}$
}

\begin{abstract}
Background: Physical activity (PA) and fruit and vegetable intake (FVI) are two key modifiable risk factors for cardiovascular disease (CVD). Achieving change in these behaviours is challenging and affected by many variables including psychosocial factors. We aimed to investigate the association between social support, stress and mood, and change in PA and FVI following provision of CVD risk information and web-based lifestyle advice.

Methods: Seven hundred sixteen blood donors (56\% male; mean age 57 years) from the intervention arms of the Information and Risk Modification (INFORM) trial, a randomised controlled trial to assess the impact of providing CVD risk and web-based lifestyle information, were analysed as a prospective cohort. We used linear and logistic regression analyses to quantify the association between social support, stress and mood at baseline and behaviour change following the intervention. We modelled objective (average acceleration measured by Axivity AX3 wrist-worn accelerometers and plasma carotenoid levels) and subjective (self-reported recreational PA and FVI) outcomes as change between baseline and 12 weeks follow-up.

Results: There was no clear association between social support and change in objective or subjective PA. Higher levels of stress and, to a lesser extent, depression symptoms were associated with smaller improvement in self-reported PA ( $\beta-1.53 \mathrm{~h} /$ week vigorous PA, 95\% confidence interval (Cl) -2.30 to $-0.75, p<0.001$ for stress; $\beta-1.64 \mathrm{~h} /$ week, $95 \% \mathrm{Cl}-3.50$ to $0.21, p=0.082$ for little interest). Higher social support was associated with greater odds and higher stress was associated with lower odds of increasing self-reported FVI to five portions per day (odds ratio (OR) $1.33,95 \% \mathrm{Cl} 1.05$ to $1.69, p=0.020$ for social support; OR $0.57,95 \% \mathrm{Cl} 0.43$ to $0.76, p<0.001$ for stress). The associations between psychosocial factors and objective FVI were not statistically significant.

Conclusions: High stress and low mood may reduce the likelihood and extent of reported change in PA and FVI following CVD risk information and advice. Greater social support may be associated with increased FVI. The role of psychosocial factors should be considered when developing, tailoring and evaluating future interventions.

Trial registration: Current Controlled Trials ISRCTN17721237. Registered 12 January 2015.

Keywords: Behaviour change, Cardiovascular disease, Fruit and vegetable intake, Physical activity, Psychosocial factor, Randomised controlled trial, Risk
\end{abstract}

\footnotetext{
*Correspondence: rl423@medschl.cam.ac.uk

${ }^{1}$ Primary Care Unit, Department of Public Health and Primary Care, University

of Cambridge, Cambridge CB2 OSR, UK

Full list of author information is available at the end of the article
}

(c) The Author(s). 2018 Open Access This article is distributed under the terms of the Creative Commons Attribution 4.0 International License (http://creativecommons.org/licenses/by/4.0/), which permits unrestricted use, distribution, and reproduction in any medium, provided you give appropriate credit to the original author(s) and the source, provide a link to the Creative Commons license, and indicate if changes were made. The Creative Commons Public Domain Dedication waiver (http://creativecommons.org/publicdomain/zero/1.0/) applies to the data made available in this article, unless otherwise stated. 


\section{Background}

Cardiovascular disease (CVD) is the leading global cause of mortality and morbidity, causing an estimated 17.7 million (31\%) deaths in 2015 [1]. Risk of CVD is influenced by many aspects of lifestyle including diet and physical activity (PA). There is evidence that populations are eating less healthy diets and becoming more sedentary [2]. Small lifestyle changes in large numbers of people have the potential to reduce the incidence of CVD risk in the population, consequently promoting behaviour change is a public health priority. Provision of CVD risk information and lifestyle advice is advocated by international guidelines and has been offered to people aged 40 to 74 years in England since 2009 through the National Health Service Health Checks programme [3, 4]. However, while provision of risk information has been shown to increase the accuracy of CVD risk perception, there is no evidence that it reduces CVD incidence [5].

There is, however, strong evidence for the health benefits of PA and high fruit and vegetable intake (FVI), including lower risks of premature mortality and CVD [6, 7]. Despite this, recent Health Survey for England data show that only $5 \%$ of individuals meet the national PA guidelines in the UK (measured by accelerometers; when self-reported PA is assessed, this is closer to 40\%) and only a quarter of people report that they meet the target consumption of five portions of fruit and vegetables per day (5 A Day) [8]. Changes have not been observed in this population over recent years [9], therefore increasing PA and FVI remains a priority in reducing the risk of CVD.

Sustained changes in behaviour are difficult to achieve. As demonstrated in models such as the behaviour change wheel [10], behaviour is subject to a range of influences including psychosocial factors. Social support describes the supportive behaviours provided by other people and the relationships that one experiences [11]. It has been found to correlate with levels of PA in both men and women and across different types and intensities of PA [12]. Anderson-Bill reported that participants in a web-based intervention with support from their friends and family were more likely to set goals and monitor their behaviour [13]. Similarly, relatives of CVD patients with greater social support had greater adherence to interventions to promote PA and healthier diet [14, 15]. Conversely, other studies have found no clear positive association between social support and change in PA following interventions, with either subjective [16] or objective measures of outcome [17]. One literature review reported that social support predicted FVI in adults [18] whereas a similar review that only included studies based on social cognitive theories did not find this association [19].

There is also considerable evidence to suggest that higher levels of stress are associated with less exercise and PA, and also that PA can reduce stress [20]. Conversely, two reviews assessing the association between psychosocial factors and FVI have not reported an association with stress $[18,19]$, although less healthy foods are consumed when stressed [21], and sugary and fatty foods can alleviate stress in some circumstances [22].

Similarly, there is a bidirectional inverse association between low mood and exercise: less PA is associated with a higher likelihood of depressive symptoms [23] and depression is consistently associated with declining levels of PA [24]. While the association between depression and FVI is perhaps less consistent and well documented, evidence suggests that FVI is inversely related to risk of depression [25] and is lower in people with depression [26]. In addition, there is some evidence that participants with depression are less likely to initiate or sustain a lifestyle behaviour change regime [27].

Evidence for the role of social support, stress and experience of low mood in determining levels of PA and FVI therefore suggests that they could influence the intention or ability to change lifestyle behaviour. However, the effect of these psychosocial factors on change in lifestyle behaviours following provision of risk information and lifestyle advice is inconclusive. We aimed to assess the association between psychosocial factors (social support, stress and mood) and short-term change in objectively measured and self-reported PA and FVI following provision of CVD risk and lifestyle behavioural change information.

\section{Methods}

\section{The INFORM trial}

The INFORM (Information and Risk Modification) trial was a parallel-group, open randomised controlled trial in England that sought to quantify the short term effects of providing information about CVD risk and lifestyle advice on health-related behaviours; the protocol has been reported previously [28]. A convenience sample of 932 participants recruited from the INTERVAL blood donation trial [29] was randomised 1:1:1:1, with stratification by age (older or younger than 60 years) and sex, to one of the following trial arms: control with no intervention; web-based lifestyle advice only; lifestyle advice plus phenotypic coronary heart disease (CHD) risk score information; or lifestyle advice plus phenotypic and genetic $\mathrm{CHD}$ risk score information. For this analysis, the control group was excluded in order to assess the association between psychosocial factors and behaviour change in people who received the intervention.

\section{Exposures and outcomes}

Social support was measured as part of a baseline questionnaire using the previously validated 12-item Multidimensional Scale of Perceived Social Support (MSPSS) 
that covers three dimensions: social support from family, friends and a significant other [30]. We excluded participants if more than half the responses from one of the dimensions were missing and calculated a mean score between one and seven; higher scores indicated greater perceived social support. Participants were also asked, "How would you rate your overall stress level?" on a five-item Likert scale from very low (one) to very high (five), based on the question used by Knowles [31]. To assess mood, two questions were adapted from the previously validated two-item Patient Health Questionnaire (PHQ-2) [32]. Participants responded 'yes' or 'no' to having often been bothered by feeling down, depressed or hopeless, and by having little interest or pleasure in doing things (anhedonia) during the previous month.

PA was measured at baseline and after three months using an Axivity AX3 3-Axis Logging Accelerometer ${ }^{\circ}$ worn continuously on the wrist for seven days. We excluded participants who wore the accelerometer for less than $24 \mathrm{~h}$ and adjusted the acceleration output for wear time. Participants were also asked to report their recreational activity, based on the previously validated European Prospective Investigation into Cancer and Nutrition (EPIC)-Norfolk Physical Activity Questionnaire [33] to estimate time typically spent per week during the past three months walking, cycling, gardening, and doing DIY, physical exercise and housework. Total self-reported PA was calculated in addition to vigorous PA (walking, cycling and exercising). If total PA was greater than $100 \mathrm{~h}$ per week, responses were recoded as missing since this could be assumed to be an error.

Total plasma carotenoid levels were assessed at baseline and follow-up by blood samples as an objective proxy measure of FVI. In addition, participants were asked how many servings of fruit and vegetables they consume on an average day (none, one, two to three, four, or five or more per day). These data were analysed as whether or not participants who failed to eat five portions per day at baseline reported doing so at follow-up.

\section{Statistical analyses}

All statistical analyses were performed using STATA 14.2. Baseline characteristics were summarised as mean and standard deviation (mean $\pm \mathrm{SD}$ ) or number and percentage of participants, as appropriate. Where data were available at baseline and follow-up, we used paired two-sample $t$ tests, Wilcoxon signed-rank tests or Pearson's chi-squared tests to test for a significant difference.

We investigated the relationships between social support, stress and mood, and change in PA and FVI between baseline and follow-up using linear regression for continuous outcomes and logistic regression for binary outcomes. Models adjusted for baseline values of the outcome (unadjusted models) and also for age, sex, randomisation group, level of income, level of education, marital status and type of occupation at baseline (adjusted models) are presented. Adjusted models are referred to in the text unless otherwise stated and $p=0.05$ is used to define statistical significance. Adjusted linear regression models were tested for normal distribution and homoscedasticity of residuals.

We also conducted sensitivity analyses where data below the 5th and above the 95th percentiles were replaced with the values of the 5th and 95th percentiles, respectively, for continuous outcomes. Sensitivity analyses for the total population and also stratified by sex are shown. We used Wilcoxon rank sum tests to test whether there was a difference between those with and without missing data for objective outcomes at either baseline or follow-up, with regards to demographics and exposures.

\section{Results \\ Participants}

The main findings from the INFORM trial of the effect of provision of risk information alongside web-based lifestyle advice on objectively measured levels of PA and FVI will be published separately. Table 1 shows the baseline characteristics of the 716 blood donors included in this analysis, which excluded the control arm. 56\% were male and the mean age was 57 years. Over half of participants had a university education (57\%) and an annual income below $£ 40,000$ (63\%). $74 \%$ were married or cohabiting and $98 \%$ classified their ethnicity as white. Most had a sedentary job (52\%) although a substantial number reported not working at present (29\%). There were similar numbers in each arm of the trial (238 or 239 participants).

Table 1 also shows that, on average, participants reported perceiving strong social support at baseline; overall mean score was $5.6 \pm 1.1 \mathrm{SD}$ (range one to seven). Participants found greatest support in their significant other and least in their friends. Most participants reported a low or moderate level of stress, although 94 participants (13\%) were very stressed. 133 participants (19\%) were bothered by feeling down, depressed, or hopeless during the past month, and 101 participants (14\%) had anhedonia. Both low mood symptoms were reported by 67 participants (9\%).

Participants on average had little increase in their objectively measured PA over the study period (Table 2): average acceleration increased from $13.0 \pm 5.6 \mathrm{SD}$ to $13.5 \pm 6.8 \mathrm{SD} \mathrm{mg} / \mathrm{min} \quad(p=0.738)$. However, self-reported total PA increased by an average $50 \mathrm{~min}$ to $20.3 \pm 12.7$ SD hours per week $(p=0.003)$, despite average self-reported vigorous PA decreasing over the study period. Objective FVI, measured by plasma carotenoids, decreased between baseline and follow-up from $3.22 \pm$ 
Table 1 Baseline demographics and psychosocial factors of participants in the INFORM trial

\begin{tabular}{|c|c|c|}
\hline & $\mathrm{N}$ & $\mathrm{n}(\%)$ or mean $\pm \mathrm{SD}$ \\
\hline \multicolumn{3}{|l|}{ Demographics } \\
\hline Sex (male) & 716 & $399(55.7)$ \\
\hline Age (years) & 716 & $56.8 \pm 8.8$ \\
\hline Education (university) & 716 & $408(57.0)$ \\
\hline Income (less than $£ 40,000$ per year) & 653 & $409(62.6)$ \\
\hline Married or living as married & 716 & $530(74.0)$ \\
\hline Ethnicity (White - British, Irish or other) & 643 & $630(98.0)$ \\
\hline Occupation category & 716 & \\
\hline Sedentary & & $370(51.7)$ \\
\hline Do not work at present & & $208(29.1)$ \\
\hline Randomisation group & 716 & \\
\hline (1) Lifestyle advice & & $239(33.4)$ \\
\hline (2) Lifestyle + phenotype & & $239(33.4)$ \\
\hline (3) Lifestyle + phenotype + genotype & & $238(33.2)$ \\
\hline \multicolumn{3}{|l|}{ Social support } \\
\hline Overall & 693 & $5.61 \pm 1.13$ \\
\hline Family & 696 & $5.55 \pm 1.34$ \\
\hline Friends & 697 & $5.45 \pm 1.27$ \\
\hline Significant other & 694 & $5.82 \pm 1.47$ \\
\hline Stress level & 710 & \\
\hline Low & & $296(41.7)$ \\
\hline Moderate & & $320(45.1)$ \\
\hline High & & $94(13.2)$ \\
\hline \multicolumn{3}{|l|}{ Mood } \\
\hline Feeling down & 710 & $133(18.7)$ \\
\hline Anhedonia & 707 & $101(14.3)$ \\
\hline
\end{tabular}

Number and percentage of participants are presented for categorical variables; mean and standard deviation are presented for continuous variables $N / n$ number of participants, $S D$ standard deviation

Randomisation groups (1) lifestyle advice only; (2) lifestyle advice plus phenotypic CHD risk score information; (3) lifestyle advice plus phenotypic and genetic CHD risk score information

'Feeling down' refers to the question "During the past month, have you often been bothered by feeling down, depressed, or hopeless?" 'Anhedonia' refers to the question "During the past month, have you often been bothered by having little interest or pleasure in doing things?"

1.44 SD $\mu \mathrm{mol} / \mathrm{l}$ to $2.74 \pm 1.15 \mathrm{SD} \mu \mathrm{mol} / \mathrm{l}(p<0.001)$. Conversely, the number of participants reporting that they eat 5 A Day increased from 218 (31\%) to 313 (50\%, $p<0.001)$, representing 133 people who increased and 19 who decreased consumption and were re-categorised.

There were few missing data overall and few significant differences in characteristics of participants with and without missing data for the objective outcomes at either baseline or follow-up (see Additional file 1: Table S1). There were 28 participants $(4 \%)$ at baseline and 92 participants (13\%) at follow-up without accelerometry data plus 46 participants $(6 \%)$ at baseline and 104 participants $(15 \%)$ at follow-up without carotenoid measurements. Those missing data were similar in terms of demographics to those with data, except that participants missing objective FVI were younger than those with data (57.1 verses 55.4 years, $p=0.045)$. Participants with missing outcome data tended to perceive lower social support, but this was only statistically significant when considering their significant other (mean score 5.9 verses 5.6 for PA data, $p=0.037 ; 5.9$ verses 5.6 for FVI data, $p=0.011$ ) and not their overall social support.

\section{Association between psychosocial factors and change in PA (Table 3)}

No association was observed between psychosocial factors and change in objective PA. Conversely, stress and low mood symptoms were negatively associated with change in self-reported PA. Per unit higher level of stress, change in vigorous PA was $1.5 \mathrm{~h}$ per week smaller (95\% CI -2.3 to $-0.8 \mathrm{~h}$ per week, $p<0.001$ ) and change in total PA was $1.4 \mathrm{~h}$ per week smaller $(95 \% \mathrm{CI}-2.4$ to -0.4 h per week, $p=0.006)$. Anhedonia appeared to have a larger impact than feeling down, with $1.6 \mathrm{~h}$ per week less change in vigorous PA reported for participants who had anhedonia compared to $0.7 \mathrm{~h}$ less in those who were feeling down (95\% CI -3.5 to $0.2, p=0.082$ and $95 \% \mathrm{CI}$ -2.4 to $0.9, p=0.383$ ). However, all of these associations between mood and change in self-reported PA were weaker and not statistically significant when adjusted for potential confounders. These associations tended to remain but were weaker in the sensitivity analysis, with the exception of anhedonia, which was significantly associated with change in objective PA in the opposite direction to that observed for subjective PA and particularly in males (see Additional file 1: Table S2).

Furthermore, Additional file 1: Table S2 shows that there were only minor differences between males and females in associations between psychosocial factors and change in PA overall. An exception is noted: the association between stress and change in reported vigorous PA was much greater in males than in females $(-1.2 \mathrm{~h}$ per week in males, $95 \% \mathrm{CI}-2.0$ to $-0.5, p=0.002 ;-0.3 \mathrm{~h}$ per week in females, $95 \%$ CI -1.1 to $0.6, p=0.533$ ). In addition, despite uncertainty, low mood tended to have stronger associations with change in PA among females, and social support may have had a more positive association with reported vigorous $\mathrm{PA}$ in males.

\section{Association between psychosocial factors and change in FVI (Table 4)}

As for objective PA, no association was observed between psychosocial factors and change in objective FVI levels. No effect of excluding extreme data was observed for change in carotenoids (see Additional file 1: Table S3). Anhedonia was associated with a smaller 
Table 2 Physical activity and fruit and vegetable intake in the INFORM trial at baseline and follow-up

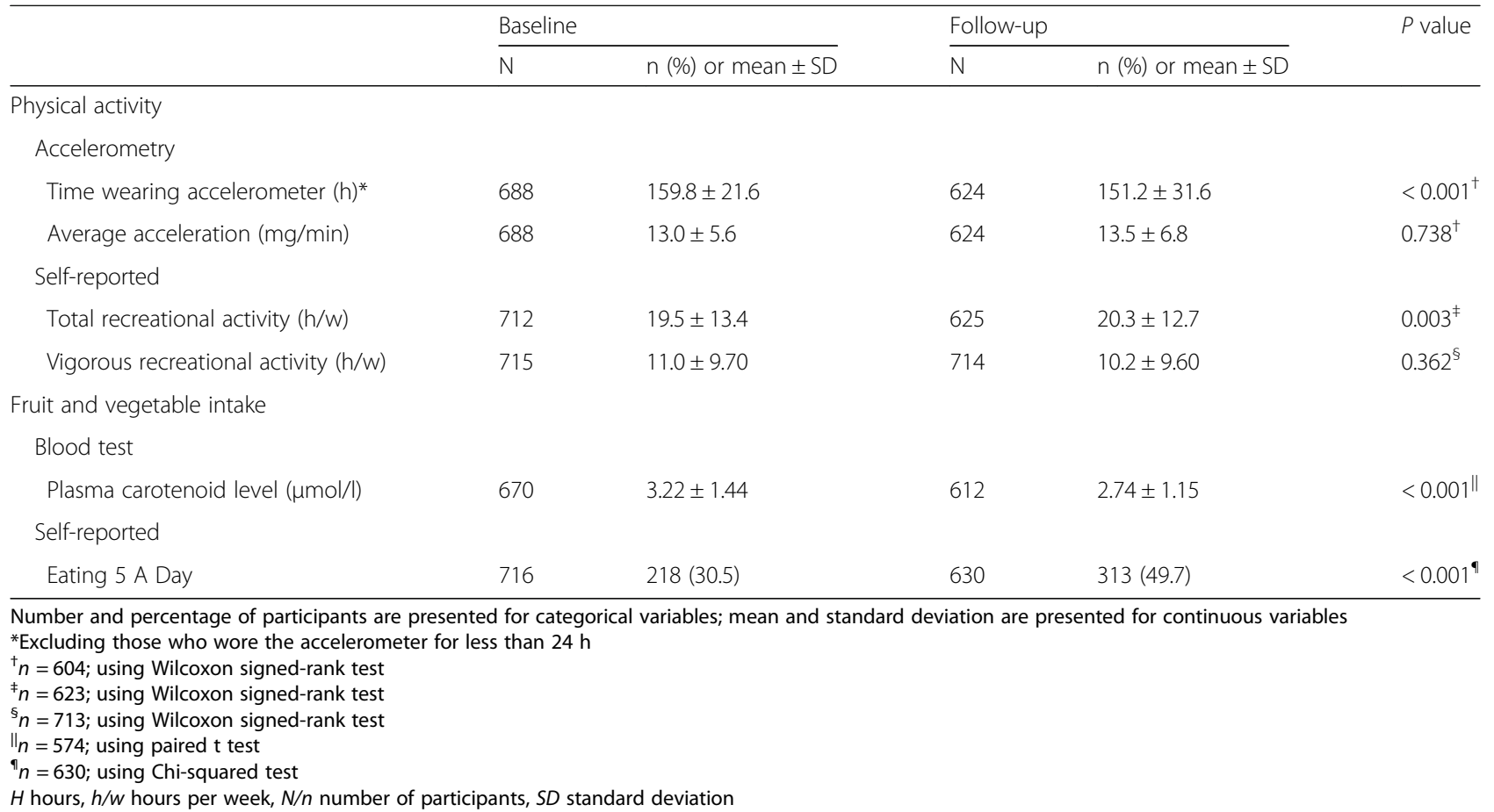

change in total carotenoid intake in males $(0.3 \mu \mathrm{mol} / \mathrm{l}$ less, $95 \%$ CI -0.5 to $-0.1, p=0.013$ ) but not in females. A similar association was seen in those feeling down, but was not significant.

Large associations were seen when considering change in the reported number of portions of fruit and vegetables consumed. For each unit increase in social support, a 1.3 times greater likelihood of increasing FVI to 5 A Day (95\% CI 1.1 to $1.7, p=0.020$ ) was observed. Stratification by sex shows that this was driven by the strong association between higher social support and greater FVI in females (Additional file 1: Table S3). Each unit increase in stress was associated with nearly half the odds of increasing to 5 A Day (OR 0.6, 95\% CI 0.4 to $0.8, p<0.001$ ). Again, the association was much stronger in females, who were 0.4 times less likely to eat $5 \mathrm{~A}$ Day per unit increase in stress (95\% CI 0.2 to $0.6, p<0.001$ ).

Similarly, reporting symptoms of low mood almost halved the odds of reporting increased FVI, and having anhedonia had a stronger association than feeling down. The strength of these associations was reduced by adjusting for potential confounders (OR 0.7, 95\% CI 0.4 to $1.3, p=0.246$ for feeling down; OR $0.6,95 \%$ CI 0.3 to $1.1, p=0.095$ for anhedonia). The association with mood symptoms again tended to be greater in females when subjective outcomes were considered.

\section{Discussion}

To our knowledge, this is the first analysis of the association between psychosocial factors and change in both objective and subjective PA and FVI outcomes. When self-reported behaviour change was considered, consistent evidence was found for the role of stress in reducing behaviour change, some evidence for mood (where experiencing anhedonia had a greater negative effect than feeling down) and some evidence for an association between social support and FVI. Sometimes the association with change in objective outcomes was in the same direction but none were statistically significant. Overall, there tended to be stronger associations between these psychosocial factors and change in self-reported FVI than PA and in females than males.

One of the strengths of this study is the inclusion of both objective and subjective outcomes. The use of objective measures overcomes issues of reporting bias due to recall error and social desirability to report healthy behaviours, giving a more accurate quantification of the association [34, 35]. The self-reported outcomes provide insight into participants' subjective views of their behaviour, which is more in line with self-monitoring after the study. A further strength is the low loss to follow-up (15\% or less) therefore low risk of attrition bias, and that any differences between those with and without missing outcome data were small.

On the other hand, the generalisability of our findings is limited because our study population consisted of research-active blood donors who are known to have better health and higher socioeconomic status than the general population [36, 37]. Baseline psychosocial factors of participants appear to be comparable to those of the 
Table 3 The association between psychosocial factors and change in physical activity outcomes

\begin{tabular}{|c|c|c|c|c|c|c|}
\hline & \multicolumn{3}{|c|}{ Unadjusted } & \multicolumn{3}{|c|}{ Adjusted } \\
\hline & $\mathrm{N}$ & $\beta(95 \% \mathrm{Cl})$ & $P$ value & $\mathrm{N}$ & $\beta(95 \% \mathrm{Cl})$ & $P$ value \\
\hline \multicolumn{7}{|l|}{ Social support } \\
\hline \multicolumn{7}{|l|}{ Objective } \\
\hline Acceleration (mg/min) & 588 & $-0.37(-0.83$ to 0.09$)$ & 0.111 & 538 & $-0.29(-0.83$ to 0.24$)$ & 0.283 \\
\hline \multicolumn{7}{|l|}{ Self-report } \\
\hline Total physical activity (h/w) & 608 & $-0.27(-1.02$ to 0.48$)$ & 0.475 & 560 & $0.03(-0.78$ to 0.84$)$ & 0.945 \\
\hline Vigorous physical activity (h/w) & 690 & $-0.58(-1.16$ to 0.00$)$ & 0.051 & 631 & $0.09(-0.53$ to 0.71$)$ & 0.778 \\
\hline \multicolumn{7}{|l|}{ Stress } \\
\hline \multicolumn{7}{|l|}{ Objective } \\
\hline Acceleration (mg/min) & 599 & $0.19(-0.37$ to 0.76$)$ & 0.501 & 547 & 0.09 (-0.58 to 0.76$)$ & 0.795 \\
\hline \multicolumn{7}{|l|}{ Self-report } \\
\hline Total physical activity (h/w) & 619 & $-1.89(-2.82$ to -0.96$)$ & $<0.001$ & 569 & $-1.42(-2.44$ to -0.41$)$ & 0.006 \\
\hline Vigorous physical activity (h/w) & 707 & $-1.73(-2.46$ to -1.00$)$ & $<0.001$ & 645 & $-1.53(-2.30$ to -0.75$)$ & $<0.001$ \\
\hline \multicolumn{7}{|l|}{ Mood (feeling down) } \\
\hline \multicolumn{7}{|l|}{ Objective } \\
\hline Acceleration (mg/min) & 598 & $0.08(-1.22$ to 1.38$)$ & 0.903 & 546 & $-0.06(-1.49$ to 1.37$)$ & 0.935 \\
\hline \multicolumn{7}{|l|}{ Self-report } \\
\hline Total physical activity (h/w) & 618 & $-2.40(-4.54$ to -0.26$)$ & 0.028 & 568 & $-1.34(-3.53$ to 0.85$)$ & 0.230 \\
\hline Vigorous physical activity (h/w) & 707 & $-1.33(-3.01$ to 0.35$)$ & 0.120 & 645 & $-0.74(-2.42$ to 0.93$)$ & 0.383 \\
\hline \multicolumn{7}{|l|}{ Mood (anhedonia) } \\
\hline \multicolumn{7}{|l|}{ Objective } \\
\hline Acceleration (mg/min) & 597 & $0.93(-0.34$ to 2.20$)$ & 0.152 & 545 & $1.03(-0.36$ to 2.41$)$ & 0.146 \\
\hline \multicolumn{7}{|l|}{ Self-report } \\
\hline Total physical activity (h/w) & 618 & $-2.43(-4.84$ to -0.03$)$ & 0.048 & 568 & $-1.49(-3.95$ to 0.97$)$ & 0.235 \\
\hline Vigorous physical activity (h/w) & 704 & $-2.16(-4.02$ to -0.29$)$ & 0.024 & 643 & $-1.64(-3.50$ to 0.21$)$ & 0.082 \\
\hline
\end{tabular}

Assessed by multiple linear regression, reporting beta coefficients. Vigorous physical activity includes walking, cycling and sport. All models are adjusted for baseline PA; the adjusted model is also adjusted for age, sex, randomisation group, marital status, income level, education level and occupation type 95\% Cl 95\% confidence interval, $h / w$ hours per week

general population, while slightly more met the 5 A Day target at baseline and are likely to be more active than the general population $[8,36]$. This may explain some of the observed lack of intervention effect because PA and fruit and vegetable consumption may already be a part of participants' usual lifestyle, which gives them less capacity for improvement and encouragement to change following receipt of CVD risk information and lifestyle advice. Consequently, the associations presented here may be conservative estimates; alternatively, unhealthier populations may be less motivated to change following receipt of risk information and lifestyle advice.

In addition, the study had a short follow-up of three months meaning that only conclusions about the association of psychosocial factors with short term lifestyle behaviour change can be made. Residual confounding by variables not considered in the design or analysis may also explain some of the associations observed and the 95\% statistical significance level should be interpreted with caution due to multiple hypotheses being tested. Moreover, a crude, yes/no form of the PHQ-2 was used (it more commonly asks how frequently the two symptoms are experienced) that may have been answered differently depending on whether someone felt depressed while completing the questionnaire. Nevertheless, it is a simple measure to detect the direction of effect and has been found to be comparable to other depression screening questionnaires [38]. It is also important to note that vigorous self-reported PA and plasma carotenoid levels decreased in the whole cohort over the study period, although we are still able to assess the association between psychosocial factors and change in these outcomes (such as whether these factors were associated with a smaller decrease).

Previous studies investigating the association between social support and healthy behaviours have been inconsistent, with some reporting an association [12-15, 18], and others not $[16,17,19]$. These studies have been in 
Table 4 The association between psychosocial factors and change in fruit and vegetable intake outcomes

\begin{tabular}{|c|c|c|c|c|c|c|}
\hline & \multirow[b]{2}{*}{ N } & \multicolumn{2}{|l|}{ Unadjusted } & \multicolumn{3}{|c|}{ Adjusted } \\
\hline & & $\beta / O R(95 \% \mathrm{Cl})$ & $P$ value & $\mathrm{N}$ & $\beta / O R(95 \% \mathrm{Cl})$ & $P$ value \\
\hline \multicolumn{7}{|l|}{ Social support } \\
\hline \multicolumn{7}{|l|}{ Objective } \\
\hline Total carotenoids $(\mu \mathrm{mol} / \mathrm{l})$ & 562 & $-0.03(-0.09$ to 0.03$)$ & 0.353 & 515 & $-0.04(-0.10$ to 0.03$)$ & 0.235 \\
\hline \multicolumn{7}{|l|}{ Self-report } \\
\hline Increase to 5 A Day & 419 & 1.39 (1.12 to 1.72$)$ & 0.002 & 391 & 1.33 (1.05 to 1.69$)$ & 0.020 \\
\hline \multicolumn{7}{|l|}{ Stress } \\
\hline \multicolumn{7}{|l|}{ Objective } \\
\hline Total carotenoids $(\mu \mathrm{mol} / \mathrm{l})$ & 570 & $0.02(-0.05$ to 0.09$)$ & 0.617 & 521 & $0.02(-0.06$ to 0.10$)$ & 0.619 \\
\hline \multicolumn{7}{|l|}{ Self-report } \\
\hline Increase to 5 A Day & 428 & 0.55 (0.43 to 0.71$)$ & $<0.001$ & 399 & 0.57 (0.43 to 0.76$)$ & $<0.001$ \\
\hline \multicolumn{7}{|l|}{ Mood (feeling down) } \\
\hline \multicolumn{7}{|l|}{ Objective } \\
\hline Total carotenoids $(\mu \mathrm{mol} / \mathrm{l})$ & 569 & $-0.04(-0.21$ to 0.12$)$ & 0.604 & 520 & $-0.05(-0.22$ to 0.13$)$ & 0.600 \\
\hline \multicolumn{7}{|l|}{ Self-report } \\
\hline Increase to 5 A Day & 427 & 0.56 (0.32 to 0.99$)$ & 0.045 & 398 & 0.70 (0.38 to 1.28$)$ & 0.246 \\
\hline \multicolumn{7}{|l|}{ Mood (anhedonia) } \\
\hline \multicolumn{7}{|l|}{ Objective } \\
\hline Total carotenoids ( $\mu \mathrm{mol} / \mathrm{l})$ & 567 & $-0.10(-0.29$ to 0.10$)$ & 0.320 & 519 & $-0.12(-0.32$ to 0.08$)$ & 0.247 \\
\hline \multicolumn{7}{|l|}{ Self-report } \\
\hline Increase to 5 A Day & 427 & 0.44 (0.23 to 0.85$)$ & 0.014 & 398 & $0.55(0.28$ to 1.11$)$ & 0.095 \\
\hline
\end{tabular}

Assessed by multiple linear regression, reporting beta coefficients for objective outcomes; or logistic regression, reporting odds ratios for self-reported outcomes. The linear regression model is adjusted for baseline carotenoid level; adjusted models are also for age, sex, randomisation group, marital status, income level, education level and occupation type

95\% Cl 95\% confidence interval, OR odds ratio

similar populations to ours, that is, in high income countries and populations without CVD. The different findings may reflect heterogeneity in the intervention, such as using a nurse-led counselling intervention [16] or the study design [13]. Our study is a simple before and after evaluation of a web-based intervention in a community setting. Furthermore, there is less literature on the role of stress and mood in behaviour change, but our findings are consistent with the what is known about the determinants of behaviour: higher levels of stress lead to less activity and consumption of unhealthy food [20-22, 39]. Similarly, depression is associated with less engagement with healthful behaviour and behaviour change programmes $[24,26,27]$.

Many of the associations were only observed in self-reported PA or FVI. The objective models may reflect the reality of very little association between psychosocial factors and lifestyle behaviour change in this cohort. Conversely, the smaller, less heterogeneous objective change in these behaviours could have attenuated associations with psychosocial factors, in comparison to the subjective outcomes. Alternatively, the associations in subjective outcomes could be due to differences in how questionnaires are completed according to level of stress or low mood, affecting accuracy of recall or engagement. For example, it is possible that all participants experienced social desirability pressure and exaggerated their recording of PA and FVI to be healthier, particularly at follow-up, because they knew that the trial aimed to influence behaviour. Both high and low exposure groups would have experienced this social desirability bias, but those experiencing stress or depression may be more inclined to record desirable outcomes in practice. It is also recognised that social desirability bias is stronger in women [40], in line with our finding of stronger associations of psychosocial factors in females.

The effect sizes observed for self-reported outcomes were large, such as 100 min lower change in vigorous PA per week for each unit increase in stress and around half the likelihood of increasing FVI when experiencing stress and low mood. If these reflect true changes in behaviour, these associations are likely to have an observable impact on daily lifestyle, with downstream impacts on CVD risk. Recent meta-analyses show that increasing PA from inactive to meeting PA guidelines is associated with 23\% lower CVD mortality [41] and that each 
additional portion of fruit or vegetables consumed every day is associated with $4 \%$ lower CVD mortality [42]. However, the association with psychosocial factors may have been exaggerated by the self-report measures, although it was sometimes supported by consistency in direction of association with objective measures.

While the longer term associations require further research, these findings are important for those developing and evaluating future lifestyle behaviour change interventions. For example, interventions may be more effective if they include additional aspects to encourage perseverance in the programme during stress, enable counselling alongside behaviour change in people with low mood, or highlight the benefits of healthy behaviours on stress and depression. Similarly, women in particular could be encouraged to discuss their plans for increasing FVI with others. The discrepancies observed in this study between objective and subjective outcomes across groups with different psychosocial characteristics also highlight the need to consider the role of these psychosocial factors when planning and interpreting research using subjective measures.

\section{Conclusion}

We have shown that high stress, low mood and lack of social support may reduce reported behaviour change in response to an intervention giving CVD risk information and lifestyle advice, particularly among women. The role of psychosocial factors should be considered when developing, tailoring and evaluating future interventions.

\section{Additional file}

Additional file 1: Table S1. Baseline demographics and psychosocial factors of participants in the INFORM trial, by those with missing data for objective outcomes at baseline or follow-up. Table S2. The association between psychosocial factors and change in physical activity outcomes for the total population and stratified by sex (sensitivity analysis). Table S3. The association between psychosocial factors and change in fruit and vegetable intake outcomes for the total population and stratified by sex (sensitivity analysis). (PDF $549 \mathrm{~kb}$ )

\section{Abbreviations}

CHD: Coronary Heart Disease; Cl: Confidence Interval; CVD: Cardiovascular Disease; FVI: Fruit and Vegetable Intake; MSPSS: Multidimensional Scale of Perceived Social Support; OR: Odds Ratio; PA: Physical Activity; PHQ-2: Twoitem Patient Health Questionnaire; SD: Standard Deviation

\section{Acknowledgements}

A complete list of investigators and contributors to the INFORM study and INTERVAL trial are provided in reference $[28,29]$.

NHS Blood and Transplant, the sponsor of the INTERVAL trial, assisted with the involvement of blood donors in INFORM. The web-based lifestyle intervention for CHD prevention is based on one that was originally developed for the Heart to Health study [43]. In addition, some materials were originally developed by Leicester Diabetes Centre. Affymetrix (Santa Clara, US) provided genotyping services. The Cambridge Medical Research Council Epidemiology Unit Physical Activity Team provided expertise. UK Biocentre (Stockport, UK) provided laboratory support. Vitas (Oslo, Norway) conducted assays. We would like to thank the Patient and Public Involvement representatives
Kathryn Lawrence and Chris Girling for reviewing study documents. Lastly, we would like to thank to all participants who agreed to take part in the INFORM trial.

\section{Funding}

The INFORM trial was funded by European Commission Framework 7 EPICCVD Grant agreement no: 279233. NHS Blood and Transplant funded the INTERVAL trial. DNA extraction and genotyping in INTERVAL/INFORM was funded by the UK National Institute of Health Research. The coordinating team for INTERVAL/INFORM at the Cardiovascular Epidemiology Unit of the University of Cambridge was supported by core funding from: UK Medical Research Council (G0800270), British Heart Foundation (SP/09/002), British Heart Foundation Cambridge Cardiovascular Centre of Excellence, and UK National Institute for Health Research Cambridge Biomedical Research Centre.

SG is a National Institute of Health Research Senior Investigator and member of the NIHR School for Primary Care Research. The University of Cambridge has received salary support in respect of SG from the NHS in the East of England through the Clinical Academic Reserve. JUS was funded by a National Institute for Health Clinical Lectureship and ALF was supported by the Medical Research Council (MC_UU_12015/4). The study sponsor was the University of Cambridge.

The study sponsor(s) or funder(s) had no role in the study design; in the collection, analysis, and interpretation of data; in the writing of the report; and in the decision to submit the article for publication. All researchers state that they are independent from funders.

\section{Availability of data and materials}

Pseudo-anonymised data are stored within the Cambridge MRC Epidemiology repository. Formal requests for data submitted through the Epidemiology Meta Data Access portal http://epi-meta.medschl.cam.ac.uk and formal requests for access will be considered via a data sharing agreement that indicates the criteria for data access and conditions for research use and will incorporate privacy and confidentiality standards to ensure data security.

\section{Authors' contributions}

The original question was conceived by all of the authors. The analysis was designed and conducted by RD with supervision from $\mathrm{AF}$, and interpreted by all authors. The manuscript was written by RD with support and critical review from AF, JUS and SG. All authors read and approved the final manuscript and agree to be accountable for all aspects of this work.

Ethics approval and consent to participate

Ethical approval was received from the National Research Ethics Service Committee East of England - Cambridge Central (14/EE/1164) on 3

December 2014. All participants provided online written informed consent. We did not provide any incentives for participation in this study.

\section{Competing interests}

The authors declare that they have no competing interests.

\section{Publisher's Note}

Springer Nature remains neutral with regard to jurisdictional claims in published maps and institutional affiliations.

\section{Author details}

${ }^{1}$ Primary Care Unit, Department of Public Health and Primary Care, University of Cambridge, Cambridge CB2 OSR, UK. ${ }^{2}$ MRC Epidemiology Unit, Institute of Metabolic Science, University of Cambridge, Cambridge CB2 OSL, UK.

Received: 26 January 2018 Accepted: 1 June 2018

Published online: 13 June 2018

\section{References}

1. World Health Organization. Cardiovascular diseases fact sheet. May 2017. Available from: http://www.who.int/en/news-room/fact-sheets/detail/ cardiovascular-diseases-(cvds). Accessed 21 July 2017.

2. Levenson JW, Skerrett PJ, Gaziano JM. Reducing the global burden of cardiovascular disease: the role of risk factors. Prev Cardiol. 2002;5:188-99. 
3. National Institute for Health Care and Excellence Guidance and guidelines Cardiovascular disease: Risk assessment and reduction, including lipid modification. Available from: www.nice.org.uk/guidance/cg181/chapter/1recommendations. Last updated: Sept 2016.

4. Goff DC, Lloyd-Jones DM, Bennett G, Coady S, D'Agostino RB, Gibbons R, et al. 2013 ACC/AHA guideline on the assessment of cardiovascular risk: A report of the Am Coll Cardiol/American Heart Association Task Force on Practice Guidelines. J Am Coll Cardiol. 2014;63:2935-59.

5. Collins DRJ, Tompson AC, Onakpoya IJ, Roberts N, Ward AM, Heneghan CJ. Global cardiovascular risk assessment in the primary prevention of cardiovascular disease in adults: systematic review of systematic reviews. BMJ Open. 2017;7:e013650

6. Aune D, Giovannucci E, Boffetta P, Fadnes LT, Keum NN, Norat T, et al. Fruit and vegetable intake and the risk of cardiovascular disease, total cancer and all-cause mortality: a systematic review and dose-response meta-analysis of prospective studies. Int J Epidemiol. 2017;46:1029-56.

7. US Department of Health and Human Services. 2008 Physical activity guidelines for Americans. President's Council on Physical Fitness and Sports Research Digest. 2008. Available from: www.health.gov/paguidelines. Date Accessed 28 June 2017.

8. Craig R, Mindell J, Hirani V. Health survey for England 2008: physical activity and fitness. NHS Information Centre; 2009.

9. NHS Digital. Health Survey for England 2015: Trend tables. Available from: https://digital.nhs.uk/catalogue/pub22616. Date Accessed 14 Nov 2017.

10. Michie S, van Stralen MM, West R, Grimshaw J, Shirran L, Thomas R, et al. The behaviour change wheel: a new method for characterising and designing behaviour change interventions. Implement Sci. 2011;6:42.

11. Allison MJ, Keller C. Physical activity maintenance in elders with cardiac problems. Geriatr Nurs. 2000;21:200-3.

12. Wendel-Vos W, Droomers M, Kremers S, Brug J, Van Lenthe F. Potential environmental determinants of physical activity in adults: a systematic review. Obes Rev. 2007:8:425-40.

13. Anderson-Bill ES, Winett RA, Wojcik JR. Social cognitive determinants of nutrition and physical activity among web-health users enrolling in an online intervention: the influence of social support, self-efficacy, outcome expectations, and self-regulation. J Med Internet Res. 2011;13:e28,

14. Aggarwal B, Liao M, Mosca L. Predictors of physical activity at 1 year in a randomized controlled trial of family members of patients with cardiovascular disease. J Cardiovasc Nurs. 2011;25:444-9.

15. Aggarwal B, Liao M, Allegrante JP, Mosca L. Low social support level is associated with non-adherence to diet at 1 year in the family intervention trial for heart health (FIT heart). J Nutr Educ Behav. 2010;42:380-8.

16. Steptoe A, Rink E, Kerry S. Psychosocial predictors of changes in physical activity in overweight sedentary adults following counseling in primary care. Prev Med. 2000;31:183-94.

17. Landry AS, Thomson JL, Madson MB, Zoellner JM, Mohn RS, Noble J, et al. Psychosocial constructs and postintervention changes in physical activity and dietary outcomes in a lifestyle intervention, Hub City steps, 2010. Prev Chronic Dis. 2015:12:140525.

18. Shaikh AR, Yaroch AL, Nebeling L, Yeh M-C, Resnicow K. Psychosocial predictors of fruit and vegetable consumption in adults a review of the literature. Am J Prev Med. 2008;34:535-43.

19. Guillaumie L, Godin G, Vézina-Im L. Psychosocial determinants of fruit and vegetable intake in adult population: a systematic review. Int J Behav Nutr Phys Act. 2010;7:12

20. Stults-Kolehmainen MA, Sinha R. The effects of stress on physical activity and exercise. Sport Med. 2014:44:81-121.

21. Zellner DA, Loaiza S, Gonzalez Z, Pita J, Morales J, Pecora D, et al. Food selection changes under stress. Physiol Behav. 2006:87:789-93.

22. Gibson EL. Emotional influences on food choice: sensory, physiological and psychological pathways. Physiol Behav. 2006;89:53-61.

23. Teychenne M, Ball K, Salmon J. Physical activity and likelihood of depression in adults: a review. Prev Med. 2008:46:397-411.

24. Roshanaei-Moghaddam B, Katon WJ, Russo J. The longitudinal effects of depression on physical activity. Gen Hosp Psychiatry. 2009;31:306-15.

25. Liu X, Yan Y, Li F, Zhang D. Fruit and vegetable consumption and the risk of depression: a meta-analysis. Nutrition. 2016;32:296-302.

26. Payne ME, Steck SE, George RR, Steffens DC. Fruit, vegetable, and antioxidant intakes are lower in older adults with depression. J Acad Nutr Diet. 2012:112:2022-7.
27. Murray J, Craigs CL, Hill KM, Honey S, House A. A systematic review of patient reported factors associated with uptake and completion of cardiovascular lifestyle behaviour change. BMC Cardiovasc Disord. 2012;12:120.

28. Silarova B, Lucas J, Butterworth AS, Di Angelantonio E, Girling C, Lawrence $\mathrm{K}$, et al. Information and risk modification trial (INFORM): design of a randomised controlled trial of communicating different types of information about coronary heart disease risk, alongside lifestyle advice, to achieve change in health-related behaviour. BMC Public Health. 2015;15:868.

29. Di Angelantonio E, Thompson SG, Kaptoge S, Moore C, Walker M, Armitage J, et al. Efficiency and safety of varying the frequency of whole blood donation (INTERVAL): a randomised trial of 45000 donors. Lancet. 2017;390: 2360-71.

30. Zimet GD, Powell SS, Farley GK, Werkman S, Berkoff KA. Psychometric characteristics of the multidimensional scale of perceived social support. J Pers Assess. 1990:55:610-7.

31. Knowles JW, Assimes TL, Kiernan M, Pavlovic A, Goldstein BA, Yank V, et al Randomized trial of personal genomics for preventive cardiology: design and challenges. Circ Cardiovasc Genet. 2012;5:368-76.

32. Kroenke K, Spitzer RL, Williams JBW. The patient health Questionnaire-2: validity of a two-item depression screener. Med Care. 2003;41:1284-92.

33. Wareham NJ, Jakes RW, Rennie KL, Schuit J, Mitchell J, Hennings S, et al. Validity and repeatability of a simple index derived from the short physical activity questionnaire used in the European prospective investigation into Cancer and nutrition (EPIC) study. Public Health Nutr. 2003;6:407-13.

34. Reilly JJ, Penpraze V, Hislop J, Davies G, Grant S, Paton JY. Objective measurement of physical activity and sedentary behaviour: review with new data. Arch Dis Child. 2008;93:614-9.

35. Hagstromer M, Ainsworth BE, Oja P, Sjostrom M. Comparison of a subjective and an objective measure of physical activity in a population sample. J Phys Act Health. 2010;7:541-50.

36. Golding J, Northstone K, Miller LL, Smith GD, Pembrey M. Differences between blood donors and a population sample: implications for casecontrol studies. Int J Epidemiol. 2013:42:1145-56.

37. Atsma F, Veldhuizen I, De Vegt F, Doggen C, De Kort W. Cardiovascular and demographic characteristics in whole blood and plasma donors: results from the donor InSight study. Transfusion. 2011;51:412-20.

38. Whooley MA, Avins AL, Miranda J, Browner WS. Case-finding instruments for depression: two questions are as good as many. J Gen Intern Med. 1997;12: 439-45.

39. Oliver G, Wardle J. Perceived effects of stress on food choice. Physiol Behav. 1999:66:511-5.

40. Dalton D, Ortegren M. Gender differences in ethics research: the importance of controlling for the social desirability response bias. Source J Bus Ethics. 2011:103:73-93.

41. Wahid A, Manek N, Nichols M, Kelly P, Foster C, Webster P, et al. Quantifying the association between physical activity and cardiovascular disease and diabetes: a systematic review and meta-analysis. J Am Heart Assoc. 2016;5: e002495.

42. Wang X, Ouyang Y, Liu J, Zhu M, Zhao G, Bao W, et al. Fruit and vegetable consumption and mortality from all causes, cardiovascular disease, and cancer: systematic review and dose-response meta-analysis of prospective cohort studies. Br Med J. 2014;349:94490.

43. Keyserling TC, Sheridan SL, Draeger LB. UNC Center for Health Promotion and Disease Prevention. 2017. Heart to Health. Available from: http://hpdp. unc.edu/research/projects/heart-to-health. Date Accessed 07 Oct 2010.

\section{Ready to submit your research? Choose BMC and benefit from:}

- fast, convenient online submission

- thorough peer review by experienced researchers in your field

- rapid publication on acceptance

- support for research data, including large and complex data types

- gold Open Access which fosters wider collaboration and increased citations

- maximum visibility for your research: over $100 \mathrm{M}$ website views per year

At BMC, research is always in progress.

Learn more biomedcentral.com/submissions 